\title{
TERAPIA OCUPACIONAL Y PACIENTE CRÍTICO
}

\author{
OCCUPATIONAL THERAPY AND CRITICAL ILL PATIENTS
}

\section{Francisca Celis D. ${ }^{1}$, Carolina Gálvez G. ${ }^{2}$, Christian Moretti A. ${ }^{3}$, Erna Navarrete ${ }^{4}$, Maximiliano Rovegno E. ${ }^{5}$ y Valentina Torrent M. ${ }^{6}$}

\section{Resumen:}

El presente estudio fue realizado en la Unidad de Pacientes Críticos adultos, del Hospital Clínico de la Pontificia Universidad Católica de Chile (UPC-HCPUC), durante el año 2012, donde se exploraron las características del paciente crítico, para obtener un perfil de salud global durante su estadía en la UPC, con el fin de determinar si sería posible realizar una intervención desde la Terapia Ocupacional que fuese un aporte a esta unidad.

Se realizó un estudio prospectivo, observacional en la UPC médico-quirúrgica durante 25 días. Los resultados obtenidos permitieron caracterizar al paciente crítico de esta unidad, como un sujeto con alta probabilidad de presentar compromiso de conciencia, edema en mano, limitación de rango de movimiento articular (ROM) en muñeca y dedos, y carencia de estímulos que evoquen su realidad previa a la hospitalización.

Finalmente, a partir del análisis del perfil del paciente crítico de la UPC-HCPUC y del contexto al que se encuentra expuesto, se concluye que la intervención temprana de Terapia Ocupacional podría disminuir y prevenir la aparición de algunos signos asociados al paciente crítico, comprobándose la hipótesis, que dadas las características de este paciente, sería posible realizar una intervención desde la Terapia Ocupacional.

\section{Palabras clave:}

Paciente crítico, Terapia Ocupacional, Unidad de Pacientes Críticos.

\begin{abstract}
:
This study was conducted in The Unit Critics adult patients, Clinical Hospital of the Catholic University of Chile (UPC- HCPUC), in 2012, where the characteristics of critical patients were explored to obtain a profile of global health while in the UPC, in order to determine whether it would be possible to make an intervention from occupational therapy to be a contribution to this unit. A prospective, observational study in medical UPC-surgery for 25 days. The results allowed to characterize critical patients of this unit, as a subject with high probability of impairment of consciousness, edema in hand, limitation of joint range of motion (ROM) in the wrist and fingers, and lack of stimuli that evoke your reality prior to hospitalization.

Finally, from the analysis of the profile of critical patient - HCPUC UPC and the context to which it is exposed, it is concluded that early intervention occupational therapy could reduce and prevent the appearance of certain signs associated with critical patient, checking hypothesized that given the characteristics of this patient, it would be an intervention from Occupational Therapy .
\end{abstract}

\section{Keywords :}

Critical Patient, Occupational Therapy, Unit of Critical Patients .

1. Licenciada en Ciencias de la Ocupación, Terapeuta Ocupacional de la Universidad de Chile. franciscacelisd@gmail.com

2. Licenciada en Ciencias de la Ocupación, Terapeuta Ocupacional de la Universidad de Chile. carolina.galvez@hotmail.cl

3. Licenciado en Ciencias de la Ocupación, Terapeuta Ocupacional de la Universidad de Chile. Docente Universidad Autónoma de Chile, sede Temuco. moretti.anfossi@hotmail.com

4. Terapeuta Ocupacional, Master in Medical Sciences in Occupational Therapy, Licenciada en Ciencia de la Ocupación Humana. Profesor Asistente en docencia. Escuela de Terapia Ocupacional Universidad de Chile. Independencia 1027, Santiago. +56229786587, enavarre@med.uchile.cl

5. Médico especialista en Medicina Interna y Medicina Intensiva, Candidato a Doctor en Ciencias Médicas. ICU «Hospital Clínico de la Pontifi cia Universidad Católica de Chile». PUC, Chile.

6. Licenciada en Ciencias de la Ocupación, Terapeuta Ocupacional de la Universidad de Chile. valentina.torrent@gmail.com 


\section{INTRODUCCIÓN}

Los servicios de salud en Chile son responsables de la articulación, gestión y desarrollo de una red asistencial correspondiente, para la ejecución de las acciones integradas de fomento, protección y recuperación de la salud, como también de la rehabilitación y cuidados paliativos (Minsal 2012).

A nivel nacional, y específicamente en Santiago, existen diversos prestadores de salud públicos y privados. Entre ellos se encuentra la Red Salud UC, red de atención médica privada, que depende de la Facultad de Medicina de la Pontificia Universidad Católica de Chile, siendo a la vez un campo clínico de estudio e investigación. Esta cuenta con Centros Médicos, Centros de Especialidades, Centros de Salud Familiar, Hospitales y Clínicas a lo largo de la Región Metropolitana. Este estudio se llevó a cabo en el Intensivo de la Unidad de Paciente Crítico (UPC) del Hospital Clínico de la Pontificia Universidad Católica de Chile (UPCHCPUC), ubicada en la Comuna de Santiago, durante junio del 2012.

La UPC es un servicio de alta complejidad, cuyo objetivo es asegurar la sobrevida de aquellas personas en estado crítico de salud. Estos pacientes pueden llegar a la unidad tras un post-operatorio, por una insuficiencia respiratoria que requiere soporte ventilatorio, por inestabilidad circulatoria o shock; necesitando monitoreo constante $y / 0$ empleo de terapias especiales como el uso de drogas vasoactivas (Tomicic, 2012).

El Intensivo de la UPC-HCPUC cuenta con un equipo de profesionales compuesto por: 2 Médicos Residentes, 1 Médico especialista en Medicina Intensiva (jefe del turno), 6 Enfermeras, 12 Auxiliares de Enfermería y 2 Kinesiólogos.

La UPC-HCPUC define al paciente crítico como aquel que necesita del monitoreo y tratamiento intensivo que no puede ser entregado fuera de la misma. En estos pacientes, generalmente, no hay límites para la prolongación de la terapia que están recibiendo, permaneciendo aquí el tiempo que sea necesario para preservar su vida. Junto a ello, pueden presentar afecciones en el sistema cardiovascular, respiratorio, endocrino, desórdenes neurológicos y/o gastrointestinales, sobredosis de drogas, quirúrgicos y misceláneas (Tomicic, 2012).

Para que un paciente sea derivado a la UPC-HCPUC debe cumplir con los siguientes criterios de inclusión (Tomicic, 2012):
- Según Signos Vitales:

- $\quad$ Pulso $<40$ o $>150$ latidos por minuto.

- Presión arterial sistólica< 80 mmHg o 20 mmHg por debajo de la presión habitual del paciente.

- Presión arterial media $<60 \mathrm{mmHg}$.

- Presión arterial diastólica > 120 mmHg.

- Frecuencia respiratoria $>35$ respiraciones por minuto.

- Según valores de laboratorio considerados son:

- Sodio sérico $<110 \mathrm{mEq} / \mathrm{L}$ ó $>170 \mathrm{mEq} / \mathrm{L}$.

- Potasio sérico $<2 \mathrm{mEq} / \mathrm{L}$ ó $>7 \mathrm{mEq} / \mathrm{L}$.

- $\mathrm{PaO} 2<50$ torr $(6.67 \mathrm{kPa})$.

- $\quad \mathrm{PH}<7.1$ ó $>7.7$.

- $\quad$ Glicemia $>800 \mathrm{mg} / \mathrm{dL}$.

- $\quad$ Calcemia $>15 \mathrm{mg} / \mathrm{dL}$.

- Niveles tóxicos de drogas u otra substancia química en un paciente comprometido neurológica o hemodinámicamente.

- Desde imagenología los criterios de inclusión son:

- Hemorragia cerebrovascular.

- Contusión.

- Hemorragia subaracnoídea con alteración de la conciencia o focalidad neurológica.

- Ruptura de víscera, vejiga, hígado, várices esofágicos, útero, con inestabilidad circulatoria.

- Aneurisma disecante de la aorta.

- Por medio de la Electrocardiografía se consideran:

- Infarto del miocardio con arritmias complejas. 
- Inestabilidad hemodinámica o insuficiencia cardíaca congestiva.

- Arritmias supraventriculares con inestabilidad hemodinámica.

- Taquicardia ventricular sostenida o fibrilación ventricular.

- Bloqueo AV completo.

- $\quad$ Signos físicos de comienzo agudo son:

- Anisocoria más alteración de conciencia.

- Quemaduras mayor al $10 \%$ de la superficie corporal.

- Anuria.

- Obstrucción de la vía aérea.

- Coma.

- Status convulsivo.

- Cianosis.

- Tamponamiento cardíaco.

Independiente de la descompensación fisiológica que estos pacientes presentan, algunos autores (Needham, 2008; Claveti, 2008; Iwashyna, 2010) han demostrado en sus estudios que existe un deterioro en las destrezas de ejecución del individuo a nivel motor y cognitivo debido a la prolongada estadía en la UPC, las condiciones de este contexto y el uso de medicamentos, lo que podría interferir en el desempeño ocupacional post hospitalización.

El texto "Survivorship Will Be the Defining Challenge of Critical Care in the 21st Century" (Iwashyna, 2010), reafirma que los pacientes ingresados en la Unidad de Cuidados Intensivos ( $\mathrm{UCl}$ ), debiesen recibir desde el primer día, cuidados que apunten al mantenimiento de sus condiciones físicas y cognitivas, con el fin de prevenir un compromiso más elevado de su estado global. Si bien, el paciente crítico presenta alto riesgo vital, la detección precoz e intervención temprana son claves para el proceso de recuperación. Sin embargo, en la UPC de HCPUC, este proceso de intervención global aún no se ha llevado a cabo, ya que para el equipo que trabaja en la unidad, el principal objetivo de intervención es la sobrevida del paciente y no cuentan con un equipo de rehabilitación suficiente para trabajar en el área.

La Terapia Ocupacional es una profesión del área de la salud que interviene en los tres niveles de atención (Chaparro 2005), utilizando como herramienta y fin principal la Ocupación. El Terapeuta Ocupacional cuenta con los recursos necesarios para evaluar e intervenir aquellos aspectos que influyen en el desempeño ocupacional del individuo. Para alcanzarlo satisfactoriamente, es necesario, entre otras cosas, intervenir en la persona, sus ocupaciones y el contexto en que las desempeña (Kielhofner, 2006).

Una de las premisas predominantes de la Terapia Ocupacional, es que el contexto en el cual la persona realiza sus ocupaciones, definido como "la variedad de condiciones interrelacionadas que están dentro y rodeando al cliente" (AOTA, 2011), influye de gran manera en el nivel de participación de éste. Bajo esta premisa es que surge el interés por hacer el estudio en este contexto donde los pacientes se encuentran en estado crítico de salud y alejados de su cotidianidad.

A nivel mundial, son escasas las investigaciones que respaldan la intervención de Terapia Ocupacional en el paciente crítico (Pohlman, 2010; Sanz, 2004; Schweickert, 2009). En Chile, este campo se encuentra aún menos explorado y como consecuencia, existe una carencia de conocimiento formal al respecto.

Este trabajo exploratorio tuvo como objetivo evaluar la pertinencia de la implementación de un programa de Terapia Ocupacional en el paciente crítico de la UPCHCPUC, buscando corroborar o invalidar la hipótesis de que el paciente crítico hospitalizado en la UPC-HCPUC contaría con características abordables desde la Terapia Ocupacional, a través de técnicas y métodos aplicados por la disciplina.

\section{MÉTODO}

En este estudio se llevó a cabo una revisión bibliográfica con el fin de conocer el estado de las investigaciones atingentes al tema de exploración, tanto a nivel nacional como internacional. Este trabajo de tipo prospectivo, observacional y descriptivo, intenta explorar las características del paciente crítico hospitalizado en la UPC-HCPUC, con el propósito de obtener un perfil 
de salud ${ }^{7}$. En base a la información recopilada, a través de revisión bibliográfica, se indagó en las variables cuantitativas como: la disminución de ROM en puño, días de estadía en la UPC, presencia de ventilación mecánica, presencia de sedación, presencia de edema en manos, entre otros; que pudiesen interferir en el estado de salud global del paciente crítico, las que fueron utilizadas para generar los marcadores aplicados en esta exploración. De esta manera, analizar si desde la perspectiva de Terapia Ocupacional es posible realizar algún tipo de abordaje terapéutico.

La muestra estuvo constituida por todo paciente mayor a 15 años, que se encontraba en un estado crítico de salud, permaneciendo hospitalizado en la UPCHCPUC, entre el 3 y el 27 de julio del 2012.

Fueron excluidos todos aquellos pacientes, que por decisión propia o de sus familiares, rechazaron la observación y/o aquellos que estuviesen bajo los protocolos de aislamiento respiratorio.

A fin de respetar los principios bioéticos ${ }^{8}$ en la exploración, se solicitó la autorización del paciente y/o la familia, con el propósito de resguardar el principio de autonomía. Además, se contó con la aprobación del equipo de Enfermería y de la Enfermera de Investigación del Departamento de Intensivo del hospital, con el fin de respetar el conducto regular de la institución.

A partir del principio de la no maleficencia, se seleccionaron pautas de evaluación que fueran válidas para la investigación, y que tuvieran el menor grado de invasión para el sujeto de estudio. Para la realización del trabajo de campo, la evaluación observacional se estructuró en sesiones de tres horas, de lunes a viernes, comenzando el día 3 de julio del 2012 y finalizando el viernes 27 del mismo mes. Las visitas de observación y aplicación de pautas de evaluación fueron realizadas por los autores, quienes registraron diariamente las observaciones y resultados en una base de datos.

Las variables registradas en la base de datos, fueron:

7 El perfil de salud considera los elementos que fueron evaluados durante el trabajo de campo, considerando antecedentes generales y clínicos del paciente, sus días de estadía en la UPC, uso de ventilación mecánica y de sedación, estado de conciencia, presencia de limitación de ROM en muñeca y dedos, y de edema en manos.

8 Se define bioética como el estudio sistemático de la conducta humana en el área de las ciencias humanas y de la atención sanitaria, en cuanto se examina esta conducta a la luz de valores y principios morales (Reich, 1995).
- Antecedentes generales y clínicos del paciente.

- Días de estadía en la UPC.

- Presencia de ventilación mecánica.

- Presencia de sedación.

- Estado de conciencia.

- Presencia de limitación de ROM en muñeca y dedos.

- Presencia de edema en manos.

- Exposición a factores ambientales que pudiesen influir en los sistemas sensoriales auditivos, visuales, olfativos, y táctiles superficiales y profundos.

Los instrumentos utilizados para la recolección de datos fueron:

- Fichas clínicas donde se obtuvo información de:

- Antecedentes personales y clínicos (edad, sexo, días de hospitalización y diagnóstico).

- Presencia de ventilación mecánica.

- Presencia de sedación.

- Observación: A través de una pauta estructurada se registraron:

- Factores ambientales a los que estaba expuesto cada paciente (presencia de estímulos auditivos, visuales, olfativos, y táctiles superficiales y profundos).

- Edema en manos: Se clasificó en "Presencia" o "Ausencia" de edema, y en "No Aplica", para aquellos casos, donde, por factores contextuales de la unidad, como por uso de vendaje en miembros superiores, fue imposible hacer esta observación.

- Coma Near Scale (Rappaport, 2000): Por medio de este instrumento se evaluó la respuesta de cada paciente a distintos estímulos sensoriales, clasificando la respuesta en cinco niveles de conciencia, desde el "No Coma", hasta el "Extremo Estado de Coma". En aqueIlos casos, donde si bien el usuario quería ser partícipe de la investigación y por razones contextuales no fue 
posible llevar a cabo la pauta de evaluación, la clasificación correspondiente fue "No Aplica".

- Movilización Pasiva (Daniels, 1997): Esta técnica consiste en dirigir el movimiento de una articulación a través de una fuerza externa. Se utilizó para registrar la limitación de los rangos de movimiento articular de muñeca y puño. En aquellos pacientes, que por medidas de restricción y/o precautorias de daños secundarios, por ejemplo, implementación de vías en zona dorsal de la mano o contenciones, el criterio de clasificación fue "No Aplica".

A partir de la información recopilada, se realizó un análisis estadístico descriptivo-exploratorio. Los datos se muestran como promedios \pm desviación estándar (s) en el caso de datos continuos y porcentajes, en el caso de datos categóricos. El análisis tuvo como propósito determinar si los datos obtenidos se correlacionaban con la información obtenida a través de la revisión bibliográfica y el posible abordaje de la Terapia Ocupacional en el paciente crítico.

\section{Resultados}

Se evaluaron 36 pacientes, los cuales cumplían con los criterios de inclusión. La muestra estuvo compuesta por 23 hombres y 13 mujeres, con un promedio de edad de 56 años $\pm 15.6 \mathrm{~s}$. Se realizaron en total 15 visitas a la unidad, siendo registradas 92 camas $^{9}$ durante todo el periodo de observación, aplicándose en cada una las pautas de evaluación mencionadas. El máximo de visitas realizadas a un mismo paciente fue de 8 veces, mientras que el promedio de los días de hospitalización del total de camas fue 16 días \pm 20 s.

Al evaluar el estado de conciencia a través de la escala "Coma Near Scale" (Tabla 1), se obtuvo que, del total de las visitas realizadas, la mayoría de los pacientes se encontraban en un "Extremo Estado de Coma", mientras que la segunda mayoría, estaban en estado de "No Coma".
Tabla 1

Resultados Coma Near Scale de las camas registradas.

\begin{tabular}{|c|c|c|}
\hline Estado de Conciencia & $\begin{array}{c}\text { No de }^{\circ} \\
\text { Camas }\end{array}$ & $\%$ \\
\hline Extremo Estado de Coma (EEC) & 49 & 53,3 \\
\hline Marcado Estado de Coma (MREC) & 6 & 6,5 \\
\hline Moderado Estado de Coma (MEC) & 4 & 4,3 \\
\hline Cerca del Estado de Coma (CEC) & 12 & 13,0 \\
\hline No Coma (NC) & 19 & 20,7 \\
\hline No Aplica (N.A.) & 2 & 2,2 \\
\hline Total de camas registradas & 92 & 100,0 \\
\hline
\end{tabular}

\%: Porcentaje en relación al total de las camas registradas.

En cuanto a la valoración del edema en los pacientes (Tabla 2), se observó que en relación al total de las visitas realizadas, la mayor parte presentaba esta condición.

Tabla 2

Resultados de observación de Edema en manos por camas registradas.

\begin{tabular}{|c|c|c|}
\hline Edema & $\begin{array}{c}\mathrm{N}^{\circ} \text { de } \\
\text { Camas }\end{array}$ & $\%$ \\
\hline Presencia & 49 & 53,3 \\
\hline Ausencia & 23 & 25,0 \\
\hline No Aplica (N.A.) & 20 & 21,7 \\
\hline Total de camas registradas & 92 & 100,0 \\
\hline
\end{tabular}

\%: Porcentaje en relación al total de camas registradas.

De acuerdo a la valoración de Rango articular de movimiento (ROM) de Muñeca y Dedos (Tabla 3), se registró que, cercano a la mitad de los pacientes, presentaba algún nivel de disminución de ROM.

9 Se entiende el concepto "Cama", como cada paciente registrado por visita diaria, sin considerar que éste pueda ser evaluado más de una vez durante el periodo de observación. No se realizó seguimiento por paciente. 
Tabla 3

Resultados de observación de ROM de Muñeca y Dedos por camas registradas

\begin{tabular}{|c|c|c|}
\hline ROM Muñeca y Dedos & $\begin{array}{c}\text { N}^{\circ} \text { de } \\
\text { Camas }\end{array}$ & $\%$ \\
\hline Conservado & 31 & 33,7 \\
\hline Disminuido & 40 & 43,5 \\
\hline No Aplica (N.A.) & 21 & 22,8 \\
\hline Total de camas registradas & 92 & 100,0 \\
\hline
\end{tabular}

\%: Porcentaje en relación al total de camas registradas.

En relación al uso de ventilación mecánica (Tabla 4), la presencia de esta terapia fue significativa.

Tabla 4

Resultados Ventilación Mecánica por camas registradas

\begin{tabular}{|c|c|c|}
\hline Ventilación Mecánica & $\begin{array}{c}\text { N}^{\circ} \text { de } \\
\text { Camas }\end{array}$ & $\%$ \\
\hline Presenta & 78 & 84,8 \\
\hline No Presenta & 14 & 15,2 \\
\hline Total de camas registradas & 92 & 100,0 \\
\hline
\end{tabular}

\%: Porcentaje en relación al total de camas registradas.

Respecto a la presencia de sedación, registrado por visita (Tabla 5), la mitad de los pacientes se encontraba bajo los efectos de sedación.

Tabla 5

Resultados presencia de sedación por camas registradas.

\begin{tabular}{|c|c|c|}
\hline Sedación & $\begin{array}{c}\mathrm{N}^{\circ} \text { de } \\
\text { Camas }\end{array}$ & $\%$ \\
\hline Presenta & 50 & 54,3 \\
\hline No Presenta & 42 & 45,7 \\
\hline Total de camas registradas & 92 & 100,0 \\
\hline
\end{tabular}

\%: Porcentaje en relación al total de camas registradas.

Finalmente, al valorar aquellos Factores Ambientales a los que cada paciente estaba expuesto al realizar las visitas (Tabla 6), se detectó que la totalidad de las habitaciones contaban con estímulos propios de un ambiente intrahospitalario, como lo son la presencia de sonidos de maquinarias y procedimientos que estimulan la vía del dolor. Por el contrario, se encontró una carencia marcada de aquellos estímulos provenientes de la cotidianidad previa a la hospitalización, como fotos, texturas y aromas del exterior. Sin embargo, se observó una alta presencia de familias visitando la unidad.

Tabla 6

Resultados de presencia de Factores ambientales por camas registradas

\begin{tabular}{|c|c|}
\hline Características de las habitaciones por cama & $\%$ \\
\hline Colores fríos y cálidos. & 100 \\
\hline Reloj análogo. & 100 \\
\hline Reloj análogo visible. & 70 \\
\hline Luz natural indirecta. & 100 \\
\hline $\begin{array}{c}\text { Contaba presencia de fotos de familiares y/o elemen- } \\
\text { tos que evoquen el exterior. }\end{array}$ & 15 \\
\hline Ruidos de maquinarias hospitalarias. & 100 \\
\hline Exposición a la voz humana. & 100 \\
\hline Exposición a voz familiar. & 80 \\
\hline Visitas frecuente de familiares. & 80 \\
\hline Exposición a estímulos suaves. & 100 \\
\hline Presencia de estímulos táctiles de su cotidianidad \\
previa. & 5 \\
\hline Exposición a temperaturas cálidas. & 100 \\
\hline Exposición a estímulos dolorosos. & 100 \\
\hline
\end{tabular}

$\%$ : Porcentaje en relación al total de camas registradas.

\section{DISCUSIÓN}

El análisis de los resultados nos permitió generar un perfil preliminar del paciente crítico hospitalizado en la UPC-HCPUC. En base a lo cual, se puede decir que es un sujeto que se caracteriza por:

- Manifestar alta presencia de edema de manos (53,3\%).

- Padecer algún grado de disminución de rangos articulares de muñeca y dedos $(40 \%)$. 
- Presentar alta frecuencia de uso de ventilación mecánica $(84,8 \%)$.

- Estar sometido a sedación en el 54,3\% de los casos.

- Presentar, generalmente, un alto compromiso de conciencia (77\%), predominando el "Extremo Estado de Coma" con un 53,3\%.

- Estar expuesto a múltiples estímulos propios del ambiente intrahospitalario (100\%).

- Presentar carencia de estímulos que evoquen su realidad previa a la hospitalización (solo $15 \%$ de presencia de imágenes del exterior, $80 \%$ expuesto a estímulos propios de la familia y $5 \%$ de exposición de estímulos táctiles del exterior).

Con respecto a la presencia de edema, Pérez (2001) señala que el uso de drogas vasodilatadoras, la inmovilidad y mal posicionamiento, influyen directamente en su aparición. En la mayoría de los casos, éste, sumado a otros factores, puede ser causal de limitación de rangos de movimientos articulares. Complementario a esto, Clavetl (2008) en el artículo "Joint contracture following prolonged stay in the intensive care unit", asegura que la estadía en la $\mathrm{UCl}$ constituye un importante factor de riesgo para presentar contracturas articulares funcionalmente significativas. Esto se debe a que la inmovilidad por periodos mayores a dos semanas, puede producir una reducción de rangos articulares con la severidad suficiente para interferir en las actividades de la vida diaria, inclusive tras el alta. En este caso, los pacientes en la UPC-HCPUC se encontraban inmovilizados por un periodo promedio de 16 días, presentando altos porcentajes de edema en manos y limitación de rangos articulares en muñeca y dedos. Esto se correlaciona con los resultados de las investigaciones revisadas.

Desde el modelo biomecánico (Kielhofner, 2006), utilizado por la Terapia Ocupacional, se plantea que si la amplitud del movimiento está limitada debido a la tensión de los tejidos blandos, es beneficioso utilizar el estiramiento para reducirla; si se debe al edema, puede utilizarse compresión y posicionamiento para disminuirlo. Con el fin de prevenir deformidades, es pertinente la movilización pasiva y activa, y posicionamiento a través del uso de férulas y otros aparatos especiales para este propósito.

El artículo "Mobilizing Patients in the Intensive Care Unit", hace referencia al estudio realizado por Bolton y Young (2007), el cual demostró que el $46 \%$ de los usuarios de la $\mathrm{UCl}$ que presentaban sepsis, falla multiorgánica y/o una prolongada ventilación mecánica, manifestaban una disfunción neuromuscular asociada a la estadía en esta unidad y a la prolongada duración de la ventilación mecánica. Este mismo estudio también demostró que aquellos pacientes que al despertar del coma presentaban debilidad muscular utilizaron ventilación mecánica por más días que aquellos usuarios que despertaban sin disminución de la fuerza muscular. Lo que implica que la duración de la ventilación mecánica y la posible diminución de la fuerza muscular son directamente proporcionales. Si consideramos la alta presencia de ventilación mecánica observada en la UPC-HCPUC (84,8\%), se puede concluir que los pacientes de esta unidad podrían presentar alteraciones neuromusculares.

En cuanto a la movilización pasiva y activa en los pacientes críticos, Schweickert et al. (2009) en su artículo "Early physical and occupational therapy in mechanically ventilated, critically ill patients: a randomised controlled trial" demostraron que tanto los efectos funcionales como psicológicos de los pacientes con ventilación mecánica pueden ser mejorados a través de la terapia física y terapia ocupacional, realizadas durante las primeras fases de la enfermedad. Así estos autores apoyan la viabilidad y la seguridad de la movilización precoz en pacientes con ventilación mecánica en cuidados intensivos. A la vez, este estudio refiere que algunos de los beneficios de la intervención son la restauración de la independencia funcional, así como la reducción del delirium ${ }^{10}$.

Luego, Pohlman et al., en el año 2010, publican el estudio "Feasibility of physical and occupational therapy beginning from initiation of mechanical ventilation" que demuestra que la terapia física y terapia ocupacional mejoran los resultados funcionales y neurocognitivos, acortando, a la vez, la duración de la ventilación mecánica, lo que también comprueba la viabilidad y

\footnotetext{
10 El delirium o síndrome confusional agudo, es un trastorno que ha sido descrito según el DSM IV (1994), como un cuadro clínico de inicio agudo y curso fluctuante, el cual se caracteriza por generar alteraciones de la conciencia, de la atención y del pensamiento. Dentro de los factores modificables destaca el déficit sensorial, inmovilización, uso de medicamentos (sedantes, anticolinérgicos, polifarmacia, retirada de alcohol o drogas), enfermedades neurológicas agudas, enfermedades intercurrentes, trastorno metabólico, cirugía, ambiente clínico, dolor, angustia emocional y privación de sueño (Inouye, 1999).
} 
seguridad de la intervención temprana, puesto que evidencia que los usuarios pueden realizar actividades inmediatamente después de ser intubados.

Si bien en la UPC-HCPUC el 54,3\% de los pacientes estaban sometidos a sedación, hay evidencia que respalda que la intervención de Terapia Ocupacional en este tipo de usuario, podría ser un facilitador en el proceso de recuperación, tal como lo demuestra la investigación de Jakob y Takala (2009) quienes, en el texto "Physical and occupational therapy during sedation stops", proponen que movilizar al paciente durante los periodos sin sedación, reduce el tiempo de ventilación, del delirium y de la estancia en la UCl. Además, de demostrar que la puntuación del índice de Barthel ${ }^{11}$ en estos pacientes aumentó, lo que refleja un mayor nivel de independencia.

Por otra parte, se observó que un alto porcentaje de pacientes en la UPC-HCPUC presenta algún grado de compromiso de conciencia $(77 \%)$, lo cual podría ser abordado desde la Terapia Ocupacional, a través de métodos y técnicas, tales como la estimulación sensorial, que según el artículo "Intervenciones agudas para las lesiones cerebrales adquiridas" (Meyer, et al., 2010), pueden ayudar al paciente a salir de un estado comatoso o vegetativo, por ejemplo, después de una lesión cerebral aguda. A la vez, en este mismo artículo se exponen datos señalando que la estimulación sensorial puede mejorar los resultados clínicos, los parámetros fisiológicos y los comportamientos indicativos de un despertar del coma ${ }^{12}$.

Es interesante señalar que ya a principios de la década de 1950, investigadores del Institute for the Achievement of Human Potential (I.A.H.P.) propusieron la idea de que los programas de estimulación sensorial del medio ambiente, con una frecuencia, intensidad y duración muy superior a las reinantes en el ámbito hospitalario habitual en aquella época, podrían mejorar la velocidad y grado de recuperación del coma. Estos autores manifestaron que en los "pacientes comatosos",

11 El índice de Barthel es una pauta de evaluación que valora el nivel de independencia en las Actividades de la Vida Diaria Básicas. Se asignan puntajes dependiendo del nivel de asistencia que requiere la persona para llevar a cabo dichas actividades.

12 Meyer, M.; Lee, D.; Aubut, J.; Teasell, R.; Megyesi, J. \& Bayona N. (2010).Intervenciones agudas para las lesiones cerebrales adquiridas. Obtenido el 20 de octubre, 2012, desde la Fundación MAPFRE, sitio web: http://traumatismocraneoencefalico.com/ modulo-16.htm aunque el problema es principalmente cerebral, hay una condición de deprivación del medio ambiente que puede conducir a un mayor deterioro de los procesos intelectuales y de percepción, acompañados por cambios en la actividad eléctrica cerebral (Le Winn, 1978).

Otros autores, tales como Sanz et al., (2004), en el texto "Terapia Ocupacional en el estado vegetativo y de mínima conciencia: estimulación sensorial" destacan la necesidad de proporcionar un tratamiento asistencial a las personas en coma o en estado vegetativo más allá de los cuidados físicos y de enfermería, apostando por una intervención con un fin terapéutico más rehabilitador. Con este ánimo se han creado las Ilamadas terapias del despertar del coma, que consisten en ofrecer una estimulación externa e intensa que ayude al paciente a aumentar el nivel de alerta, lo que adquiere gran importancia en el contexto de la UPC-HCPUC antes mencionado.

Si bien el objetivo de este estudio fue la recolección de datos respecto a las características del paciente y contexto hospitalario, existe otro factor importante dentro del proceso de recuperación, la familia, quienes, durante el proceso de evaluación, visitaban constantemente la unidad, manifestando sentimientos de angustia e inseguridad por la situación que estaban viviendo. Abbasi et al. (2009) exploraron acerca de las intervenciones agudas en pacientes en coma post traumatismo craneal, utilizando la escala de Coma de Glasgow (GCS), detectando que aquellos sujetos que recibían visitas de su familia registraron aumentos significativos de las puntuaciones GCS todos los días durante el período del estudio. A partir del sexto día, las puntuaciones GCS en el grupo de intervención fueron significativamente mayores.

En relación a estos antecedentes y las nuevas circunstancias señaladas se realizó una intervención en la que se educó a la familia acerca del proceso de recuperación del paciente y cómo ellos podían ser partícipes de éste, a través, principalmente, de la estimulación sensorial y el manejo del edema. Posterior a esta intervención, se realizó una encuesta telefónica, en donde los familiares destacaron sentir mayor seguridad y más partícipes del proceso de recuperación de su familiar en estado crítico. Por todo esto, se concluyó que la familia, además de bajar sus niveles de ansiedad al ser parte del tratamiento, constituye un importante medio terapéutico.

Si bien en la práctica clínica hay terapeutas ocupacionales, que actualmente se desempeñan prestando servicios en las diversas Unidades de Pacientes Críticos en Chile, en la mayoría de los casos, su intervención y resultados no están respaldados por investigaciones 
que validen su actuar. Por esto se estima necesario fomentar la publicación de artículos que evidencien la importancia de la intervención de Terapia Ocupacional en las unidades de pacientes críticos para tener más conocimientos y validar aún más la intervención.

Por otro lado, para comprobar la efectividad de un servicio de Terapia Ocupacional en la UPC- HCPUC, es necesario asistir diariamente a la unidad, con un total de horas suficientes para intervenir a cada usuario y observar el comportamiento de cada uno según los horarios y la cantidad de sedación. Mitchell (1990) estipula que un protocolo de estimulación sensorial debería variar de uno o dos ciclos de estimulación diarios, de aproximadamente una hora cada uno, a una sesión de estimulación multimodal y una sesión de estimulación unimodal por día durante 10 minutos cada una. Wilson (1991) plantea ciclos de estimulación cada hora, de aproximadamente 15-20 minutos, mientras que Doman (1993), durante 12-14 horas por día, seis días por semana. Por esto, se cree necesario que si se desea hacer una intervención adecuada de Terapia Ocupacional en estimulación sensorial en la UPC, se debe contar con un profesional de tiempo completo destinado a estas tareas en la unidad. Además se sugiere realizar futuras investigaciones a nivel nacional, que busquen crear nuevos protocolos de estimulación sensorial y/o actualizar los ya existentes.

\section{CONCLUSIÓN}

Se realizó un trabajo exploratorio en un área compleja y con poca evidencia de respaldo, donde luego del análisis de los resultados entregados por las pautas aplicadas, y su correlación con la bibliografía existente, se puede decir que se comprueba la hipótesis de que el paciente crítico hospitalizado en la UPC-HCPUC cuenta con características abordables desde la Terapia Ocupacional, a través de técnicas y métodos aplicados por la disciplina.

Se afirma entonces, que dado el contexto y las características propias del paciente hospitalizado en la UPC-HCPUC, la intervención de Terapia Ocupacional podría ser un aporte, ya que cuenta con las bases teóricas y prácticas para ayudar a prevenir gran parte de los deterioros asociados al paciente crítico, a través de la detección precoz y la intervención temprana; asumiendo un rol que actualmente no está siendo desarrollado por el equipo profesional de la unidad. Esto cobra gran importancia en los tiempos contemporáneos, puesto que el enfoque actual de salud apunta a una atención integral, donde no solo se consideran los estándares biomédicos, sino que también, al individuo en todas sus dimensiones y contexto.

\section{AGRADECIMIENTOS}

Para finalizar, queremos agradecer la colaboración del equipo multidisciplinario de la UPC-HCPUC, quienes facilitaron el ingreso a la unidad y pusieron las instalaciones del recinto a nuestra disposición. También consideramos importante reconocer la colaboración y voluntad de los pacientes y familiares partícipes de este estudio.

Por otra parte, les damos nuestros más sinceros agradecimientos a todas aquellas personas que nos apoyaron y acompañaron durante el proceso de formación académica.

\section{ReferenCias Bibliográficas}

Ávila, A.; Martínez, R.; Matilla, R. et al. (2010). Marco de Trabajo para la práctica de la Terapia Ocupacional: Dominio y proceso, Segunda Edición. Obtenido el 22 de octubre del 2012, sitio web: http://www.terapia-ocupacional.com/aota2010esp.pdf.

Balasch, M.; Bonet, J.; Callén, B.; Guarderas, Paz. et al. (2005) Investigación Crítica: Desafíos y Posibilidades. Athenea Digital; 8: 129-144.

Burtin, C.; Clerckx, B.; Robbeets, C.; Ferdinande, P, \& Langer, D. (2009). Early exercise in critically ill patients enhances shortterm functional recovery. Crit Care Med 2009; 37(9): 24992505.

Chaparro, R., Fuentes, J.; Morán, D. et al. (2005). La Terapia Ocupacional en la intervención en salud comunitaria: el modelo educativo. Revista Chilena de Terapia Ocupacional; 5: 69-79.

Clavet, H.; Hébert, P.; Fergusson, D.; Doucette, S. \& Trudel, G. (2008). Joint contracture following prolonged stay in the intensive care unit. Canadian Medical Association or its licensors; 178 (6): 691-697.

Cid, J.; Damián, J. (2012). Valoración de la discapacidad física: el Índice de Barthel [versión electrónica]. Revista Española de Salud Pública 1997; 71(2): 127-137. Obtenido el 3 de diciembre del 2012, desde http://scielo.isciii.es/scielo.php?script=sci_arttext\& pid=S1135-57271997000200004 
Corominas, C.; Lara, R.; Manuel-Rimbau, E. \& Lozano, P. (2012). Desde Hospital Universitari son Dureta, sitio Obtenido el 11 de noviembre 2013 web: http://www.google.cl/ url? sa $=t \& r c t=j \& q=\&$ esrc $=s \&$ source $=$ web $\& c d=13 \& v e d=0$ CHQQFjAM\&url=http $\% 3 \mathrm{~A} \% 2 \mathrm{~F} \% 2 \mathrm{Fwww}$. capitulodeflebologia.org\%2Fbeca_leo\%2F2007\%2F0703151215457. doc\&ei=N1WmUMCOO8PX0QH1-YDoCw\&usg=AFQjCNEs XYiwTIKMvgpjyUz7Pp9UImOs4g\&sig2=Mlzf3_Mcv4p1uAu$\mathrm{X} 1 \mathrm{gJ} \times \mathrm{w}$

Daniels, L. \& Northingam, C. Pruebas funcionales musculares. Madrid: Editorial Interamericana, 1997.

Empaire, G.; D’Empaire, M. \& Encinoso, J. (2002). Limitación de medidas terapéuticas en los pacientes críticos. Gaceta Médica de Caracas; 110: 92-109.

Estenssoro,; E., Reina, R.; Canales, H.; Sáenz, M.; González, F. et al. (2006). The distinct clinical profile of chronically critically ill patients: a cohort study. Critical Care; 10 (3): 1-9.

Griffiths, R. \& Hall, J. Intensive care unit-acquired weakness (2010). Crit Care Med; 38(3): 779-787.

Iwashyna, T. (2010). Survivorship Will Be the Defining Challenge of Critical Care in the 21st Century. Ann Intern Med; 153: 204-205.

Jakoba, S. \& Takalaa, J. (2009). Physical and occupational therapy during sedation stops. The Lancet; 373: 1824-1826.

Kielhofner, G. (2006). Fundamentos conceptuales de la terapia ocupacional (3ra ed). Buenos Aires: Médica Panamericana.

Kortebein, P.; Ferrando, A.; Lombeida, J. \& Wolfe, R. (2007). Effect of 10 Days of Bed Rest on Skeletal Muscle in Healthy Older Adults. American Medical Association; 297(16): 1772-1774.

Martínez, C. (2012). Semiología: Edema y Cianosis. Obtenido el 11 de noviembre, 2012, desde Universidad del Nordeste, Facultad de Medicina, sitio web: http://med.unne.edu.ar/catedras/medicinai/semioclas/edeycia.pdf

Meyer, M.; Lee, D.; Aubut, J.; Teasell, R.; Megyesi, J. \& Bayona, N. (2010) Intervenciones agudas para las lesiones cerebrales adquiridas. Obtenido el 20 de octubre, 2012, desde la Fundación MAPFRE, sitio web: http://traumatismocraneoencefalico.com/ modulo-16.htm

Ministerio de Salud. Servicios de Salud (2012). Obtenido el 31 de octubre, 2012, desde Ministerio de Salud, Gobierno de Chile, sitio web: http://www.minsal.gob.cl/portal/url/page/minsalcl/g_ conozcanos/g_subs_redes_asist/servicios $\% 20-\% 20$ funciones. html.

Needham, D. (2008). Mobilizing Patients in the Intensive Care Unit Improving Neuromuscular Weakness and Physical Function. JAMA; 300 (14): 1685-1690.

Pérez, J.; Salem, C.; Henning, E.; Uherek, F. \& Schultz C, (2001). Linfedema de miembro superior secundario al tratamiento de cáncer de mama [versión electrónica]. Cuadernos de cirugía; 15 (1): 107 - 115.
Pohlman, M., Schweickert, W,; Pohlman, A.; Nigos, C.; Pawlik, A,. et al. (2010). Feasibility of physical and occupational therapy beginning from initiation of mechanical ventilation. Crit Care Med; 38 (1): 2089-2094.

Rappaport, M.; (2012). The Coma/Near Coma Scale. The Center for Outcome Measurement in Brain Injury, 2000. Obtenido el 12 de marzo, 2012, desde Guía Clínica Asistencial, Manejo del paciente con déficit prolongado del nivel de conciencia, sitio web: http://www.humv.es/index.php?option=com docman\&task=doc_view\&gid $=65 \&$ Itemid $=27$

Reich, W. Encyclopedia of Bioethics. Nueva York: Macmillan Publishing Company, 1995.

Sanz, S.; DePobes, A.; Bové, M.; Tàsies, S.; Andrés, B. et al. (2004) .Terapia Ocupacional en el estado vegetativo y de mínima conciencia: estimulación sensorial. Mapfre Medicina; 15 (2): 112-117.

Schweickert, W.; Pohlman, M.; Pohlman, A.; Nigos, C.; Pawlik, A.,et al. (2009). Early physical and occupational therapy in mechanically ventilated, critically ill patients: a randomized controlled trial. The Lancet; 373: 1874-1882.

Tomicic, V. (2012). Admisión y Alta a Unidades de Cuidados Intensivos. Obtenido el 11 de septiembre, 2012, desde la Universidad Católica de Chile, Programa de Medicina Intensiva, sitio web:http://escuela.med.puc.cl 